\title{
Cuticle darkening correlates with increased body copper content in Drosophila melanogaster
}

\author{
Johana Vásquez-Procopio (D) \\ Subhash Rajpurohit $\mathbb{D} \cdot$ Fanis Missirlis
}

Received: 2 June 2020 / Accepted: 29 August 2020 / Published online: 7 October 2020

(C) Springer Nature B.V. 2020, corrected publication 2022

\begin{abstract}
Insect epidermal cells secrete a cuticle that serves as an exoskeleton providing mechanical rigidity to each individual, but also insulation, camouflage or communication within their environment. Cuticle deposition and hardening (sclerotization) and pigment synthesis are parallel processes requiring tyrosinase activity, which depends on an unidentified copper-dependent enzyme component in Drosophila melanogaster. We determined the metallomes of fly strains selected for lighter or darker cuticles in a laboratory evolution experiment, asking whether any specific element changed in abundance in concert with pigment deposition. The results showed a correlation between total iron content and strength of pigmentation, which was further corroborated by ferritin iron quantification. To ask if the observed increase in iron body content along with increased pigment deposition could be generalizable, we crossed yellow and ebony alleles causing light and dark pigmentation, respectively, into similar genetic backgrounds and measured their metallomes. Iron remained unaffected in the various mutants providing no support for a causative link
\end{abstract}

J. Vásquez-Procopio $\cdot$ F. Missirlis $(\bowtie)$

Departamento de Fisiología, Biofísica y Neurociencias,

Cinvestav, Zacatenco, Mexico City, Mexico

e-mail: fanis@ fisio.cinvestav.mx

S. Rajpurohit

Division of Biological and Life Sciences, School of Arts and Sciences, Ahmedabad University, Commerce Six

Road, Navrangpura, Ahmedabad, Gujarat, India between pigmentation and iron content. In contrast, the combined analysis of both experiments suggested instead a correlation between pigment deposition and total copper body content, possibly due to increased demand for epidermal tyrosinase activity.

Keywords Color $\cdot$ Elemental analysis $\cdot$ Genetic background $\cdot$ Melanin $\cdot$ Zinc

\section{Introduction}

Insect cuticle is the primary interface between an insect and its environment. It is a polymer network composed of chitin, protein derivatives, and hydrocarbons (Chapman 1998). Darkening of the cuticle is mostly due to non-lipid indole and catechol components, also referred to as cuticle pigments or melanin (Hackman and Goldberg 1971). In common to other insects, the epidermal cells of Drosophila melanogaster produce cuticle pigments during the final day of metamorphosis and the first hours after adult eclosion (Wittkopp and Beldade 2009; Wright 1987). The cuticle extracellular matrix is secreted through a developmental process initiated by the ecdysis neuropeptide signaling pathway (Davis et al. 2007; Luan et al. 2006) and further controlled by physiological (Shakhmantsir et al. 2014) and genetic (Gibert et al. 2007; Ostrowski et al. 2002) cues. Cuticle pigmentation occurs when melanin is deposited directly into the epidermis (Walter et al. 1996). Two well-known 
mutants that significantly change body color in $D$. melanogaster are yellow (y) and ebony (e) (Wittkopp et al. 2002). The former cause a pale yellow body color, whereas the latter result in a dark black body color. Although the melanin deposition pathway in model organisms has been characterized in detail, the chemistry around this process is poorly understood (Massey and Wittkopp 2016). Melanin formation requires the action of a multi-enzyme complex, sometimes referred to as tyrosinase (Lewis and Lewis 1963; Wright 1987). Historically, tyrosinase activity has been studied through assays that follow the oxidation of 3,4-dihydroxyphenylalanine (dopa), a reaction that also forms a key biosynthetic step in the formation of melanin (Lewis and Lewis 1963; Walter et al. 1996). Somewhat surprisingly, the relative quantities of extractable tyrosinase activity in larvae and pupae of the $y$, wild type and $e$ genotypes increased in reverse relation to pigmentation (Graubard 1933). It should be noted, however, that the tyrosinase complex acting at the epithelial cells appears to be distinct from the prophenol oxidases that produce melanin during the immune response, given that deletion of all three prophenol oxidases rendered flies susceptible to microbial infection without altering their cuticle pigmentation (Binggeli et al. 2014; Dudzic et al. 2015). Yet, one of the enzymatic components was found to be a cuproenzyme (Seybold et al. 1975; Sugumaran et al. 1992) and, most importantly, the cuticle pigmentation activity required the presence of copper in vivo (Binks et al. 2010; Turski and Thiele 2007; Zhang et al. 2020; Zhou et al. 2003).

There is a large body of literature assessing the 'melanism-desiccation' hypothesis, which proposes that dark cuticle in several Drosophila species is an adaptation for increased desiccation tolerance (Gibbs and Rajpurohit 2010; Parkash et al. 2008; Rajpurohit et al. 2016a). Interestingly, an early report suggested that $e$ mutants of $D$. melanogaster were more resistant to desiccation stress than wildtype flies, whereas $y$ mutants of multiple species were less desiccation resistant (Kalmus 1941). In addition, $e$ mutants produce longer hydrocarbon cuticle chains (Massey et al. 2019a), whereas male $y$ mutants have difficulty to sustain copulation (Massey et al. 2019b), underscoring the pleiotropic function of these genes. It was shown independently that selection in the laboratory for desiccation resistance produced populations with longer hydrocarbon chains (Gibbs et al. 1997). In another study, laboratory selection for pigmentation darkness resulted in correlated resistance to desiccation (Ramniwas et al. 2013). Thus, it was somewhat surprising that an independent attempt to replicate these findings resulted in the opposite conclusion, namely that selection for pigmentation darkness or desiccation resistance evolved independently of each other (Rajpurohit et al. 2016a). The last two studies shared a similar experimental design, therefore their contrasting results could relate to diverse genetic backgrounds used as starting material for selection, underscoring the difficulty on reaching generalizable conclusions for the linkage between physiological traits in experimental evolution studies. Here, we measured the metallomes of the flies selected for light or dark pigment in the latter study, as well as mutant alleles for the $y$ and $e$ genes after these were crossed in two distinct genetic backgrounds, respectively. Our finding that total body copper correlates with melanin deposition is consistent with the previously shown biochemical link between this metal and cuticle pigmentation (Binks et al. 2010; Turski and Thiele 2007; Zhang et al. 2020; Zhou et al. 2003).

\section{Materials and methods}

\section{Fly strains}

The generation of fly strains with different levels of cuticle pigmentation through experimental selection during over 100 generations has been described previously in detail (Rajpurohit and Gibbs 2012; Rajpurohit et al. 2016a). The flies were maintained without selection for several months, while the present study was undertaken, but no decline in the stark differences in their pigmentation was observed, suggesting that the selection process had resulted in fixation of alleles defining cuticle pigmentation. We obtained from the Bloomington Drosophila Stock Center (BDSC) stocks Canton$S(C S ; \# 1)$ and Oregon-R (OR; \#5) from which we generated isogenic lines by performing single crosses for twenty consecutive generations. We then crossed into the respective isogenic strains a single male from BDSC stocks $y^{l}$ (\#169), $y^{2}$ (\#184), $e^{s}$ (\#498) and $e^{11}$ (\#497) and recovered the homozygous stocks for each of these alleles. We repeated this process five times to obtain the four alleles that 
provide a gradient of lighter and darker cuticle pigment, in two different genetic backgrounds. An isogenic stock from wild type strain Tan3 (Sadraie and Missirlis 2011) was also used as a control. All flies were fed on a yeast-molasses diet (Rempoulakis et al. 2014) and maintained at $25{ }^{\circ} \mathrm{C}$.

\section{Elemental analysis}

Male and female flies of the indicated genotype were collected between an age of 4 and 7 days old. The animals were freeze-dried for $8 \mathrm{~h}$ to remove water. $20 \mathrm{mg}$ of dry sample was digested in $1 \mathrm{~mL}$ of concentrated (65\%) metal-free Suprapur ${ }^{\circledR}$ nitric acid (Merck, Kenilworth, NJ, USA) at $200{ }^{\circ} \mathrm{C}$ for $15 \mathrm{~min}$ in closed vessels of the MARS6 microwave digestion system (CEM Corporation, Matthews, NC, USA). Samples were diluted with Milli-Q water to $5 \mathrm{~mL}$ and metal concentrations were measured against calibration curves and a digestion blank in a PerkinElmer Optima 8300 instrument (Shelton, CT, USA), which conducts inductively coupled plasmaoptic emission spectrometry (ICP-OES). All measurements fell within the linear range of the calibration curves, adjusted for copper, manganese between [0.02-1 ppm]; iron, zinc [0.1-5 ppm]; calcium, magnesium [0.5-25 ppm]; sodium [1-50 ppm]; potassium, phosphorus [2-100 ppm].

\section{Ferritin quantifications}

30 adult females of the indicated genotype were homogenized in $120 \mathrm{~mL}$ of ice-cold extraction buffer $(137 \mathrm{mM} \mathrm{NaCl}, 20 \mathrm{mM}$ TRIS-HCL and 1\% Triton $\mathrm{X}-100 \mathrm{pH}$ 7.5). The whole fly homogenates were centrifuged at $18,800 \times g$ for $15 \mathrm{~min}$ and the supernatant was transferred to a new tube. To remove lipids, the extract was centrifuged at $9600 \times g$ for $5 \mathrm{~min}$. The clear supernatant was recovered and assessed on nonreducing SDS-PAGE acrylamide gels stained with Coomassie and Prussian blue stains, as described previously (Rosas-Arellano et al. 2016). The gels were photographed using an ImageQuant ${ }^{\mathrm{TM}}$ LAS 400 camera system for producing digital images of gel samples. Band intensities were quantified from three biological replicates using the Image-J software and normalized as described in the results.
Statistical analysis

One-way analysis of variance (ANOVA) was performed between specified groups setting the significance cut-off value at $\mathrm{p}<0.05$ and using the GraphPad Prism 5 software. A Tukey's post-hoc test was applied to compare the different groups, where the ANOVA indicated statistically significant differences.

\section{Results}

Metallomes in strains from the directed evolution experiment

Nine strains described in detail previously (Rajpurohit and Gibbs 2012; Rajpurohit et al. 2016a) were maintained on our normal laboratory diet (Rempoulakis et al. 2014) without further selection. Observing their cuticle pigment, the flies could be readily distinguished between the light (L), control (C) and dark (D) phenotypes, suggesting the respective trait had been fixed in the population (Rajpurohit et al. 2016b). L, C and D strains each comprised of three independently selected (or, in the case of $\mathrm{C}$, separately maintained) populations (Rajpurohit et al. 2016a). Copper, manganese, iron, zinc, calcium, magnesium, sodium, potassium and phosphorus content was measured by ICP-OES in 4 to 7 days-old adult flies (Table 1). We report the average value and standard deviation from triplicate measurements performed on three biological replicates, in separately timed experiments/collections, for each of the nine strains. Given our interest to discover associations between elemental content and pigmentation, we performed statistical analysis of variance using the data collected for the L, C and D strains collectively $(n=9$ independent values per group). The L strain showed higher manganese compared to the $\mathrm{C}$ and $\mathrm{D}$ strains and lower iron compared to the $\mathrm{D}$ strain. The $\mathrm{C}$ strain showed lower calcium to both the L and D strains, and lower sodium and phosphorus compared to the D strain. The D strain showed higher zinc and magnesium compared to the $\mathrm{C}$ and $\mathrm{L}$ strains (Table 1). Copper concentrations did not change in a statistically significant manner in the three groups (Table 1 and Fig. 1a). Copper and iron changed in abundance in a consistent gradient according to pigmentation, with iron increasing from $0.09 \pm 0.02 \mathrm{mg}[\mathrm{Fe}] / \mathrm{g}$ fly body dry weight in $\mathrm{L}$ strains 
Table 1 Metallomes of strains selected for differences in pigmentation through experimental evolution

\begin{tabular}{|c|c|c|c|c|c|c|c|c|c|}
\hline & $\begin{array}{l}\mu \mathrm{g} \mathrm{Cu} / \mathrm{g} \\
\text { weight }\end{array}$ & $\begin{array}{l}\mu \mathrm{g} \mathrm{Mn} / \mathrm{g} \\
\text { weight }\end{array}$ & $\begin{array}{l}\mathrm{mg} \mathrm{Fe} / \mathrm{g} \\
\text { weight }\end{array}$ & $\begin{array}{l}\mathrm{mg} \mathrm{Zn/g} \\
\text { weight }\end{array}$ & $\begin{array}{l}\mathrm{mg} \mathrm{Ca} / \mathrm{g} \\
\text { weight }\end{array}$ & $\begin{array}{l}\mathrm{mg} \mathrm{Mg} / \mathrm{g} \\
\text { weight }\end{array}$ & $\begin{array}{l}\mathrm{mg} \mathrm{Na} / \mathrm{g} \\
\text { weight }\end{array}$ & $\begin{array}{l}\mathrm{mg} \mathrm{K} / \mathrm{g} \\
\text { weight }\end{array}$ & $\begin{array}{l}\mathrm{mg} \mathrm{P} / \mathrm{g} \\
\text { weight }\end{array}$ \\
\hline Tan3 & $12.4 \pm 2.2$ & $20.8 \pm 2.2$ & $0.09 \pm 0.01$ & $0.17 \pm 0.02$ & $0.45 \pm 0.03$ & $0.86 \pm 0.06$ & $0.78 \pm 0.09$ & $5.6 \pm 1.1$ & $7.8 \pm 0.5$ \\
\hline $\mathrm{La}$ & $12.7 \pm 0.2$ & $18.2 \pm 2.2$ & $0.08 \pm 0.01$ & $0.13 \pm 0.00$ & $0.43 \pm 0.06$ & $0.72 \pm 0.10$ & $0.70 \pm 0.09$ & $5.3 \pm 0.7$ & $6.7 \pm 1.0$ \\
\hline $\mathrm{Lb}$ & $11.5 \pm 2.2$ & $21.2 \pm 1.4$ & $0.10 \pm 0.01$ & $0.13 \pm 0.01$ & $0.46 \pm 0.03$ & $0.70 \pm 0.07$ & $0.71 \pm 0.11$ & $5.3 \pm 0.7$ & $6.8 \pm 0.6$ \\
\hline Lc & $11.8 \pm 1.8$ & $17.0 \pm 1.9$ & $0.11 \pm 0.02$ & $0.16 \pm 0.01$ & $0.42 \pm 0.08$ & $0.67 \pm 0.07$ & $0.73 \pm 0.09$ & $5.2 \pm 0.7$ & $6.9 \pm 0.7$ \\
\hline $\mathrm{L}$ & $12.0 \pm 1.5$ & $18.8 \pm 2.5^{\mathrm{a}}$ & $\mathbf{0 . 0 9} \pm 0.02^{\mathbf{a}}$ & $\mathbf{0 . 1 4} \pm 0.01^{\mathbf{a}}$ & $\mathbf{0 . 4 4} \pm 0.06$ & $\mathbf{0 . 7 0} \pm 0.07$ & $\mathbf{0 . 7 1} \pm 0.09^{\mathbf{a b}}$ & $\mathbf{5 . 3} \pm 0.6$ & $6.8 \pm 0.7^{\mathbf{a b}}$ \\
\hline $\mathrm{Ca}$ & $12.5 \pm 2.4$ & $13.6 \pm 1.1$ & $0.11 \pm 0.02$ & $0.15 \pm 0.02$ & $0.35 \pm 0.04$ & $0.67 \pm 0.06$ & $0.67 \pm 0.02$ & $5.0 \pm 0.4$ & $6.3 \pm 0.5$ \\
\hline $\mathrm{Cb}$ & $13.6 \pm 3.4$ & $14.8 \pm 1.6$ & $0.11 \pm 0.01$ & $0.13 \pm 0.01$ & $0.34 \pm 0.02$ & $0.69 \pm 0.10$ & $0.66 \pm 0.05$ & $5.1 \pm 0.7$ & $6.7 \pm 0.9$ \\
\hline $\mathrm{Cc}$ & $11.5 \pm 1.6$ & $13.1 \pm 0.9$ & $0.11 \pm 0.01$ & $0.14 \pm 0.01$ & $0.33 \pm 0.03$ & $0.66 \pm 0.06$ & $0.63 \pm 0.01$ & $4.8 \pm 0.3$ & $6.3 \pm 0.4$ \\
\hline $\mathrm{C}$ & $12.5 \pm 2.4$ & $\mathbf{1 3 . 8} \pm 1.3^{\mathbf{b}}$ & $\mathbf{0 . 1 1} \pm 0.01^{\mathbf{a b}}$ & $\mathbf{0 . 1 4} \pm 0.02^{\mathbf{a}}$ & $\mathbf{0 . 3 4} \pm 0.03^{\mathbf{a}}$ & $\mathbf{0 . 6 8} \pm 0.07$ & $0.66 \pm 0.03^{\mathbf{a}}$ & $4.9 \pm 0.5$ & $6.4 \pm 0.6^{\mathbf{a}}$ \\
\hline $\mathrm{Da}$ & $12.8 \pm 2.0$ & $16.5 \pm 0.6$ & $0.11 \pm 0.02$ & $0.16 \pm 0.01$ & $0.44 \pm 0.01$ & $0.79 \pm 0.09$ & $0.77 \pm 0.10$ & $5.3 \pm 0.7$ & $7.3 \pm 0.7$ \\
\hline $\mathrm{Db}$ & $14.0 \pm 2.1$ & $18.6 \pm 1.0$ & $0.11 \pm 0.02$ & $0.14 \pm 0.01$ & $0.48 \pm 0.06$ & $0.84 \pm 0.11$ & $0.83 \pm 0.12$ & $5.5 \pm 0.8$ & $7.8 \pm 0.8$ \\
\hline Dc & $12.5 \pm 1.1$ & $12.7 \pm 1.6$ & $0.12 \pm 0.03$ & $0.18 \pm 0.01$ & $0.43 \pm 0.03$ & $0.79 \pm 0.06$ & $0.73 \pm 0.05$ & $5.4 \pm 0.5$ & $7.5 \pm 0.6$ \\
\hline D & $13.1 \pm 1.7$ & $15.9 \pm 2.8^{\mathbf{b}}$ & $\mathbf{0 . 1 2} \pm 0.02^{\mathbf{b}}$ & $\mathbf{0 . 1 6} \pm 0.02^{\mathbf{b}}$ & $\mathbf{0 . 4 5} \pm 0.04$ & $\mathbf{0 . 8 1} \pm 0.08^{\mathbf{a}}$ & $\mathbf{0 . 7 8} \pm 0.09^{\mathbf{b}}$ & $\mathbf{5 . 4} \pm 0.6$ & $7.5 \pm 0.7^{\mathbf{b}}$ \\
\hline
\end{tabular}

A Tukey post-hoc comparison between the light (L), control (C) and dark (D) pigment groups is indicated with minor case letters (statistical difference is present when letters are different between groups). In bold font mean values and standard deviations from the mean are given for all independent measurements of the preceding groups, respectively. Letters are only included when a one-way ANOVA performed on the pool of subgroup replicate measurements identified difference between groups at $\mathrm{p}<0.05$

to $0.11 \pm 0.01 \mathrm{mg} / \mathrm{g}$ in $\mathrm{C}$ strains to $0.12 \pm 0.02 \mathrm{mg} / \mathrm{g}$ in D strains (Table 1). Although L flies were different to D flies when considered globally for iron, ANOVA between individual strains did not identify statistically significant differences (Fig. 1b).

\section{Ferritin iron determinations}

We have previously shown significant correlation between total body iron and ferritin iron in flies (Gutierrez et al. 2013; Sadraie and Missirlis 2011). We therefore isolated proteins from 4 to 7-days-old mated female flies and analyzed ferritin iron (Fig. 1c) and ferritin protein (Fig. 1d) in each of the strains. We noted a correlation whereby the L strains had least ferritin iron or protein between the three groups, whereas the D strains had most. We quantified ferritin iron through densitometric analysis (Fig. 1e). The results showed a statistically significant difference between the $\mathrm{L}_{\mathrm{A}}$ strain, which had similar ferritin iron content to the wild type Tan 3 control strain, and strains $\mathrm{D}_{\mathrm{A}}$ and $\mathrm{D}_{\mathrm{B}}$, which showed a two-fold increase in iron content compared to Tan3. To further demonstrate that the $\mathrm{L}$ flies were lower in iron than the $\mathrm{D}$ flies (controls having intermediate values), we plotted the fold difference of body iron content of each individual strain to Tan 3 against the same ratio for ferritin iron content (Fig. 1f). A linear correlation was found with an $\mathrm{R}^{2}$ value of 0.74 . Importantly, the $\mathrm{L}$ strains had the lowest ratios, the $\mathrm{C}$ strains presented intermediate values and the $\mathrm{D}$ strains showed higher values. From these data, we conclude that during the experimental selection for dark or light cuticle, D strains were also selected for higher ferritin iron content, whereas L strains showed diminished ferritin iron.

Metallomes in strains from the genetic mutation experiment

We next asked whether mutation in a single gene that alters cuticle pigment would also produce changes in the metallome. Here, we were primarily interested in testing whether the production of excess melanin would also correlate with a change in iron in flies sharing similar genetic background, bar a single mutation. We decided to work with two classical mutants, $y$ and $e$, and two different alleles for each of these, namely $y^{l}, y^{2}$ and $e^{s}, e^{11}$, respectively. The mutant alleles were first backcrossed for five generations into two different isogenic backgrounds generated from $O R$ and $C S$ wild type flies. Metallomes from the resulting stocks are presented (Table 2). Iron did not change between the $y$ mutants (L), wild type flies (C), and $e$ mutants (D) maintaining the same 

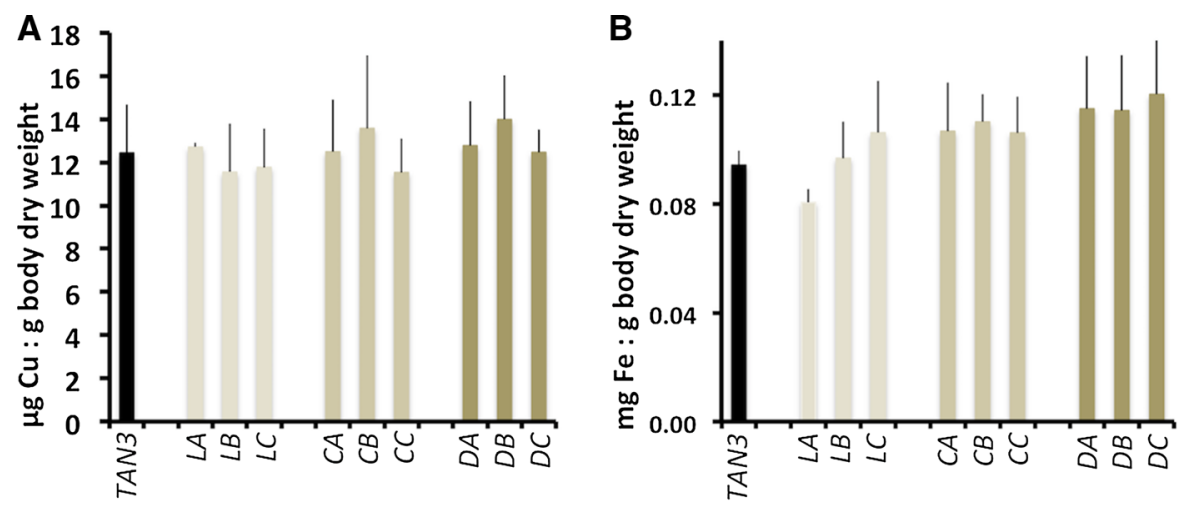

C
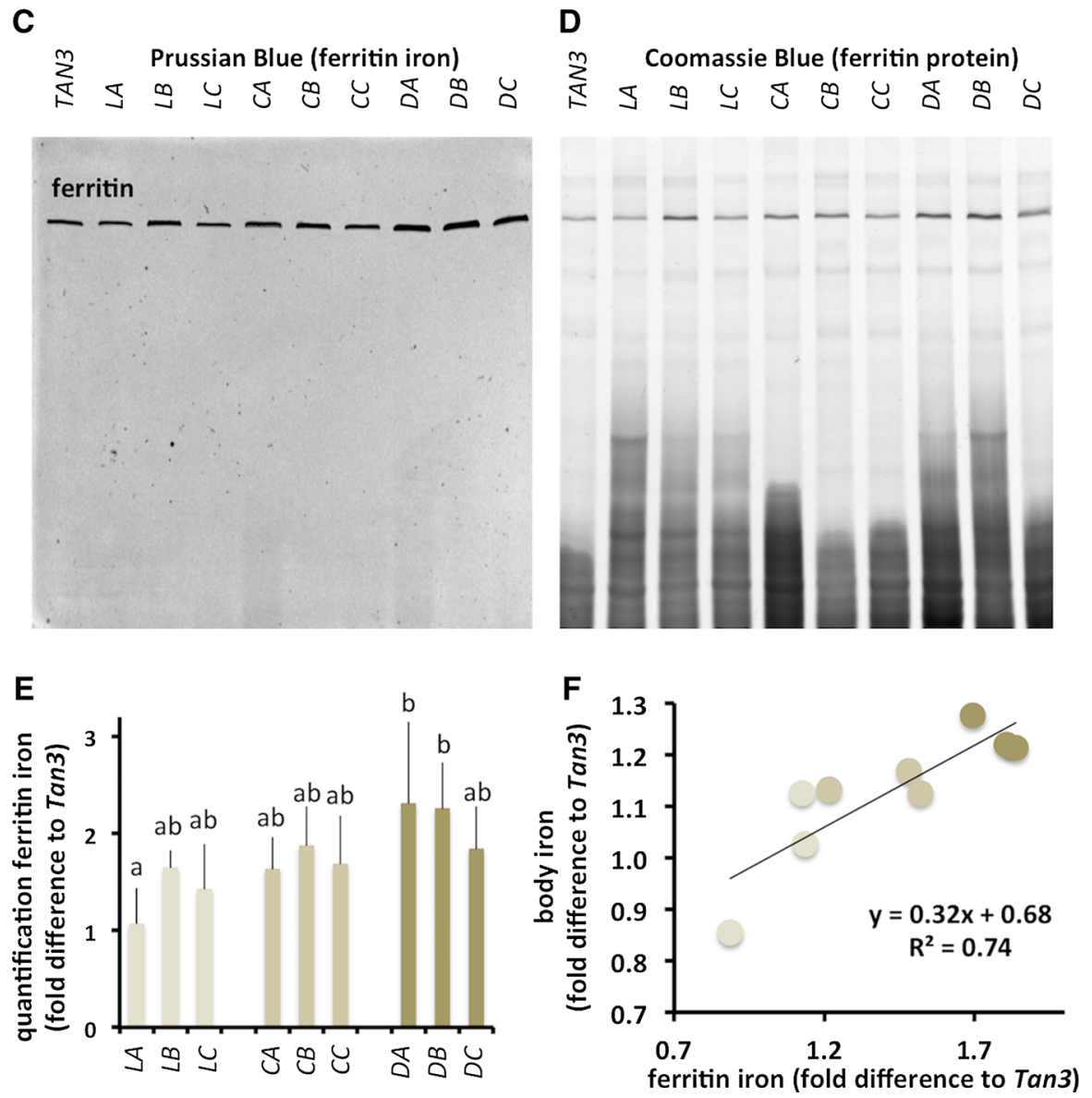

Fig. 1 Correlation between body ferritin/iron content and pigmentation strength following selection through experimental evolution. a Plot of mean values (in $\mu \mathrm{g}$ copper per g dry body weight) and standard deviations from the mean of adult flies (4-7 days old; mixed sex) in each three sub-populations of the L, C and D groups. One-way ANOVA showed no differences between the groups. b Similar plot for iron (values in $\mathrm{mg}$ iron per g dry body weight). c SDS-PAGE under non-reducing conditions followed by Prussian blue staining to reveal ferritin iron. d The same type of gel stained with Coomassie blue to reveal all proteins. The ferritin complex is readily distinguishable due to its abundance, stability and high molecular weight.

e Quantification from 3 independent experiments of ferritin iron gels. One-way ANOVA followed by a Tukey post-hoc test reported a statistically significant $(\mathrm{p}<0.05)$ difference between the $L_{A}$ and the $D_{A}$ and $D_{B}$ groups. Lower case letters indicate statistical comparisons between groups; only groups without overlapping letters are show statistical difference to each other. f The mean value for body iron as determined in (b) was normalized for each strain against the wild type TAN3 control and plotted against the values in (e). The result of regression analysis is indicated. Variations in circle color correspond to the different pigmentation intensity in the L, C and D strains 
Table 2 Metallomes of mutant alleles of $y$ and $e$ after six generations of back crossing to isogenic Canton-S and Oregon-R stocks

\begin{tabular}{|c|c|c|c|c|c|c|c|c|c|}
\hline & $\begin{array}{l}\mu \mathrm{g} \mathrm{Cu} / \mathrm{g} \\
\text { weight }\end{array}$ & $\begin{array}{l}\mu \mathrm{g} \mathrm{Mn} / \mathrm{g} \\
\text { weight }\end{array}$ & $\begin{array}{l}\mathrm{mg} \mathrm{Fe} / \mathrm{g} \\
\text { weight }\end{array}$ & $\begin{array}{l}\mathrm{mg} \mathrm{Zn/g} \\
\text { weight }\end{array}$ & $\begin{array}{l}\mathrm{mg} \mathrm{Ca} / \mathrm{g} \\
\text { weight }\end{array}$ & $\begin{array}{l}\mathrm{mg} \mathrm{Mg} / \mathrm{g} \\
\text { weight }\end{array}$ & $\begin{array}{l}\mathrm{mg} \mathrm{Na} / \mathrm{g} \\
\text { weight }\end{array}$ & $\begin{array}{l}\mathrm{mg} \mathrm{K} / \mathrm{g} \\
\text { weight }\end{array}$ & $\begin{array}{l}\mathrm{mg} \mathrm{P} / \mathrm{g} \\
\text { weight }\end{array}$ \\
\hline$C S y^{I}$ & $9.9 \pm 1.8$ & $12.5 \pm 3.9$ & $0.13 \pm 0.01$ & $0.21 \pm 0.04$ & $0.73 \pm 0.24$ & $0.92 \pm 0.07$ & $1.1 \pm 0.0$ & $6.5 \pm 0.7$ & $8.3 \pm 0.9$ \\
\hline OR $y^{l}$ & $7.7 \pm 3.3$ & $11.7 \pm 1.3$ & $0.13 \pm 0.01$ & $0.19 \pm 0.03$ & $0.58 \pm 018$ & $0.95 \pm 0.09$ & $1.1 \pm 0.1$ & $6.5 \pm 1.2$ & $8.7 \pm 0.5$ \\
\hline$C S y^{2}$ & $10.4 \pm 1.3$ & $14.0 \pm 3.7$ & $0.14 \pm 0.01$ & $0.23 \pm 0.03$ & $086 \pm 0.22$ & $0.96 \pm 0.03$ & $1.1 \pm 0.1$ & $6.6 \pm 1.0$ & $8.5 \pm 0.5$ \\
\hline$O R y^{2}$ & $8.9 \pm 2.5$ & $10.5 \pm 1.7$ & $0.13 \pm 0.00$ & $0.23 \pm 0.03$ & $0.65 \pm 0.08$ & $0.98 \pm 0.09$ & $1.2 \pm 0.0$ & $6.6 \pm 1.3$ & $8.7 \pm 0.7$ \\
\hline$L$ & $9.2 \pm 2.3$ & $12.2 \pm 2.8$ & $\mathbf{0 . 1 3} \pm 0.01$ & $0.21 \pm 0.03^{\mathbf{a}}$ & $\mathbf{0 . 7 1} \pm 0.20$ & $\mathbf{0 . 9 5} \pm 0.07$ & $\mathbf{1 . 1} \pm 0.1$ & $6.5 \pm 0.9$ & $\mathbf{8 . 6} \pm 0.6$ \\
\hline$C S$ & $11.0 \pm 1.1$ & $15.3 \pm 2.5$ & $0.14 \pm 0.01$ & $0.20 \pm 0.01$ & $1.02 \pm 0.33$ & $0.96 \pm 0.04$ & $1.2 \pm 0.1$ & $6.5 \pm 0.5$ & $8.3 \pm 0.5$ \\
\hline$O R$ & $8.6 \pm 1.7$ & $11.2 \pm 3.3$ & $0.13 \pm 0.01$ & $0.19 \pm 0.03$ & $0.81 \pm 0.19$ & $1.03 \pm 0.08$ & $1.2 \pm 0.1$ & $6.5 \pm 1.1$ & $9.2 \pm 1.1$ \\
\hline Tan3 & $10.3 \pm 2.1$ & $16.4 \pm 3.2$ & $0.12 \pm 0.01$ & $0.18 \pm 0.02$ & $0.86 \pm 0.29$ & $0.96 \pm 0.08$ & $1.0 \pm 0.2$ & $6.3 \pm 0.5$ & $8.1 \pm 0.7$ \\
\hline C & $\mathbf{1 0 . 0} \pm 1.8$ & $14.3 \pm 3.5$ & $\mathbf{0 . 1 3} \pm 0.01$ & $0.19 \pm 0.02^{\mathbf{a b}}$ & $\mathbf{0 . 9 0} \pm 0.29$ & $\mathbf{0 . 9 8} \pm 0.08$ & $1.2 \pm 0.2$ & $6.4 \pm 0.5$ & $\mathbf{8 . 5} \pm 0.7$ \\
\hline$C S e^{s}$ & $9.3 \pm 2.4$ & $7.7 \pm 1.0$ & $0.12 \pm 0.02$ & $0.18 \pm 0.03$ & $0.51 \pm 0.19$ & $0.83 \pm 0.14$ & $1.1 \pm 0.2$ & $5.9 \pm 1.4$ & $7.7 \pm 0.6$ \\
\hline$O R e^{s}$ & $12.1 \pm 8.8^{*}$ & $15.5 \pm 11.0 *$ & $0.13 \pm 0.01$ & $0.17 \pm 0.03$ & $0.90 \pm 0.21$ & $1.04 \pm 0.12$ & $1.2 \pm 0.2$ & $6.8 \pm 1.2$ & $9.0 \pm 0.2$ \\
\hline$C S e^{11}$ & $11.4 \pm 1.6$ & $14.0 \pm 5.6$ & $0.15 \pm 0.00$ & $0.20 \pm 0.02$ & $0.81 \pm 0.45$ & $0.93 \pm 0.12$ & $1.2 \pm 0.1$ & $6.5 \pm 1.3$ & $8.4 \pm 1.3$ \\
\hline OR $e^{11}$ & $9.6 \pm 2.5$ & $12.5 \pm 2.6$ & $0.13 \pm 0.00$ & $0.18 \pm 0.01$ & $0.69 \pm 0.19$ & $1.02 \pm 0.01$ & $1.3 \pm 0.1$ & $6.6 \pm 0.9$ & $8.7 \pm 0.7$ \\
\hline$D$ & $10.6 \pm 4.3$ & $12.4 \pm 6.2$ & $\mathbf{0 . 1 3} \pm 0.01$ & $\mathbf{0 . 1 8} \pm 0.03^{\mathbf{b}}$ & $\mathbf{0 . 7 3} \pm 0.28$ & $0.96 \pm 0.13$ & $1.2 \pm 0.2$ & $6.4 \pm 1.1$ & $\mathbf{8 . 4} \pm 0.9$ \\
\hline
\end{tabular}

Statistical analysis was performed as described in Table 1. In bold font mean values and standard deviations from the mean are given for all independent measurements of the preceding groups, respectively. Asterisks denote a group that showed high variance between replicate measurements for copper and manganese

value of $0.13 \pm 0.01 \mathrm{mg}[\mathrm{Fe}] / \mathrm{g}$ dry weight in all cases (Table 2). This showed that it is possible to uncouple melanin deposition in cuticle from body iron content. Zinc was the only element that showed a statistically significant difference between $y$ and $e$ flies; it was $0.21 \pm 0.03 \mathrm{mg}[\mathrm{Zn}] / \mathrm{g}$ dry weight in the $y$ mutants (L) versus $0.18 \pm 0.03 \mathrm{mg}[\mathrm{Zn}] / \mathrm{g}$ dry weight in the $e$ mutants (D). Zinc storage is associated with the metal's retention in the Malpighian tubules (TejedaGuzman et al. 2018), but we found in this experiment that $\mathrm{L}$ flies were higher in zinc content than D flies, whereas in the selection experiment the L flies were lower than D flies. Therefore it is unlikely that zinc content correlates with pigmentation more generally.

\section{Copper content correlated with pigmentation in both experiments}

The example of zinc discussed above led us to correlate the results from the two experiments described in this paper for all metals (Fig. 2). The outcome of this analysis was striking with respect to copper, where a linear regression between the two experiments gave an $\mathrm{R}^{2}$ value of 1 . Copper values for $\mathrm{L}$ flies in the first experiment were $12.0 \pm 1.5 \mu \mathrm{g}[\mathrm{Cu}] / \mathrm{g}$ dry weight versus $13.1 \pm 1.7 \mu \mathrm{g}[\mathrm{Cu}] / \mathrm{g}$ dry weight for $\mathrm{D}$ flies (Table 1), i.e. only 9\% increase. In the second experiment, L flies had $9.2 \pm 2.3 \mu \mathrm{g}[\mathrm{Cu}] / \mathrm{g}$ dry weight versus $10.6 \pm 4.3 \mu \mathrm{g}[\mathrm{Cu}] / \mathrm{g}$ dry weight for $\mathrm{D}$ flies (Table 2), which amounted to a $15 \%$ increase. Both of these results were not considered on their own statistically significant. We note, however, the consistent trend in both experiments, which is specific to copper. Iron also separated the three groups in this analysis, but the effect was fully driven from the first experiment, resulting in a flat line (Fig. 2). No other metal showed a consistent order between the L, C and D groups in this type of analysis. We therefore conclude that copper content correlated with pigmentation strength in both experiments presented here.

\section{Discussion}

Causation versus correlation in experimental evolution observations

The original study (Rajpurohit et al. 2016a) that preceded and inspired the work we described here had been performed against a considerable background of observations from fieldwork (Parkash et al. 2008) or laboratory settings (Ramniwas et al. 2013) formulating the 'melanism-desiccation' hypothesis, which suggested a functional (causal) 
Fig. 2 Correlation between body copper content and pigmentation strength in D. melanogaster. For each metal, we plotted the mean value (in $\mathrm{mg}$ [metal]/g body dry weight) for $\mathrm{L}, \mathrm{C}$ and $\mathrm{D}$ strains, respectively; on the $\mathrm{X}$-axis the result from the selection experiment and on the Y-axis the result from the mutation experiment. The intensity of coloration of the circles corresponds to the intensity of coloration of the pigments. Linear regression analysis was performed for copper and iron because these were the only metals for which L, C and D flies were distributed along the $\mathrm{X}$-axis in direct relation to their pigmentation. The positive correlation between the two experiments for copper suggests that darker flies accumulate more copper in their bodies
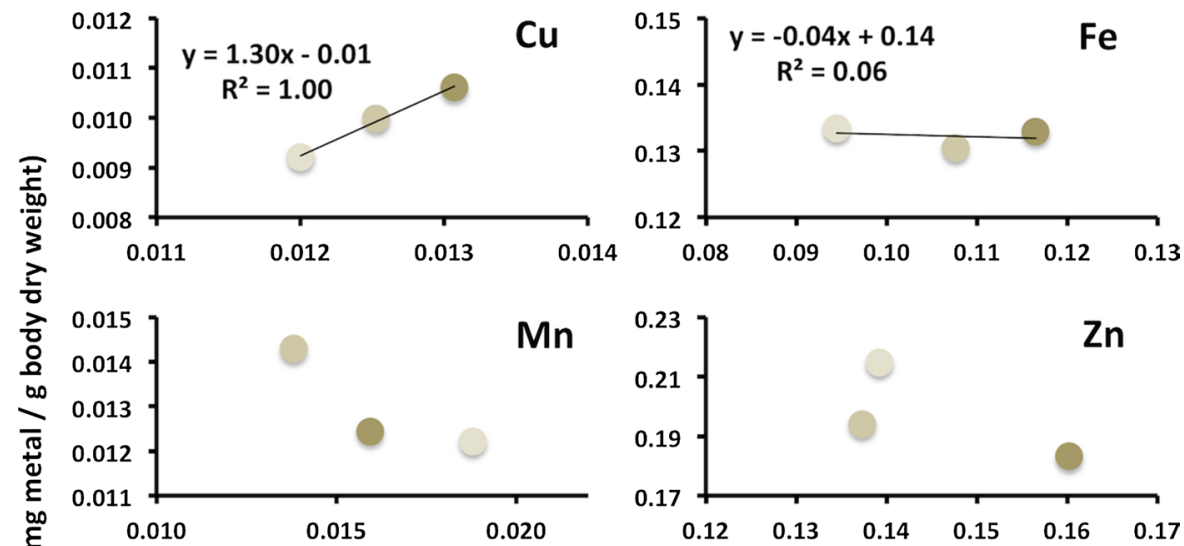

0.011

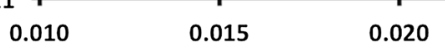

0.12

Mg
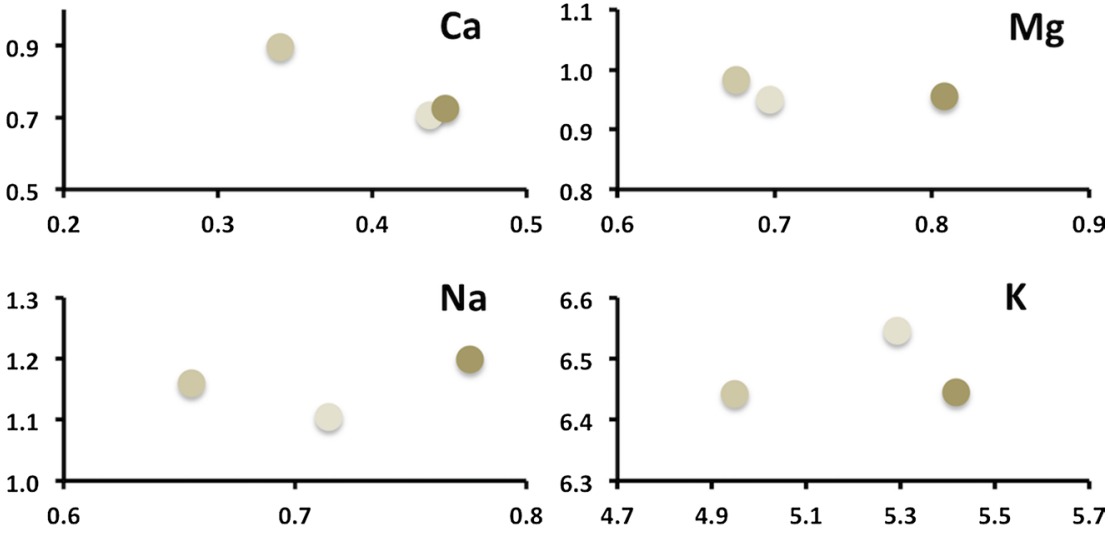

Selection experiment (mg metal / $\mathrm{g}$ body dry weight) correlation between these phenotypic traits in flies. A discussion of why in some populations two traits may be co-segregating, whereas in others not, has already been offered (Rajpurohit et al. 2016a). The argument boils down to the fact that adaptation under selective pressure takes different routes in most settings and is largely influenced by the preexisting genetic variability in any given population (Gibbs 1999; Kapun et al. 2020). In addition, pleiotropic effects of single gene mutations complicate the picture (Massey et al. 2019a). Therefore, the documentation of a clear correlation, as is for example the association of increased ferritin iron in flies with higher melanism (Fig. 1f) in populations selected on the basis of their cuticle phenotype (Rajpurohit et al. 2016a), was further interrogated in a differently controlled genetic study, based on known genes that severely affect cuticle pigmentation in D. melanogaster. In this case, the correlation was not shown to hold out (Table 2 and Fig. 2).
Correlation of metal content with specific metalloenzyme usage

Previous studies of fly metallomes have suggested a high degree of conservation in their relative concentrations between different species from the Tephritidae and Drosophilidae families (Rempoulakis et al. 2014; Sadraie and Missirlis 2011). In the case of transition metals that show high-affinity binding to proteins (zinc, iron, copper, manganese and molybdenum), these studies proposed that the predominant factor that determines how much metal is concentrated in flies is the abundance of the metalloenzymes that require the different metals. In addition to this factor, one needs to consider specific metal storage mechanisms, like the accumulation of zinc storage granules (Tejeda-Guzman et al. 2018) or, in the case of iron, of the ferritin complex (Hernandez-Gallardo and Missirlis 2020). Several studies in D. melanogaster have correlated total body iron to 
ferritin iron content (Bettedi et al. 2011; GonzalezMorales et al. 2015; Rempoulakis et al. 2014; Sadraie and Missirlis 2011; Xiao et al. 2014). This correlation is perhaps expected given that each ferritin complex concentrates 1000 of iron atoms. Recently, we made the observation that manganese accumulates rapidly in the body of young D. melanogaster adult flies of both sexes, specifically during the first 5 to 6 days of their life, after which the daily rate of increase becomes imperceptible (Vasquez-Procopio et al. 2020). During the same period, at least one manganese-dependent enzyme, superoxide dismutase was also found to increase its activity. Here, we observed correlation between copper content and pigmentation in D. melanogaster (Fig. 2). The critical reader will have noted that the data supporting this correlation are not particularly compelling; in neither of two independent sets of experiments did the differences in copper accumulation between the different groups reach statistical significance (Tables 1,2 ). We therefore rely on the strong correlation of trends in the copper measurements between the two experiments (Fig. 2). In the second experiment, the D group value could be skewed, however, by a highly variable measurement in the $O R e^{s}$ flies (Table 2). Unfortunately, all flies used in this study were disposed of during the Covid-19 contingency and we cannot, regretfully, revisit this issue on the same set of flies. With these considerations in mind, and given the known requirement of copper for pigmentation (Binks et al. 2010; Turski and Thiele 2007; Zhang et al. 2020; Zhou et al. 2003), in addition to other physiological processes (Comstra et al. 2017; Kirby et al. 2008; Sellami et al. 2012; Theotoki et al. 2019; Wang et al. 2018), we conclude this report suggesting that the observed correlation between copper content and pigmentation in D. melanogaster could be explained by an increased requirement for tyrosinase activity for melanin production in this species. To further test this hypothesis, an unambiguous identification of the copper-dependent enzyme involved in epidermal pigment melanization would be helpful (Riedel et al. 2010).

Acknowledgements We acknowledge Mexico's Consejo Nacional de Ciencia y Tecnología (Conacyt) Infrastructure Grant \#268296 in 2016, which allowed for the purchase of the ICP-OES used in this research. JVP was supported by Conacyt Ph.D. fellowship \#426510. Experiments were funded from Cinvestav's internal budget. The SR laboratory is supported through a Ramanujan Fellowship, SERB-India (SB/S2/
RJN-129/2017). We thank Fidel de la Cruz Hernández-Hernández and two anonymous reviewers for critical comments on the manuscript.

Author contributions JVP and FM performed the experiments. SR produced the "experimental evolution" set of flies. JVP, SR and FM wrote the manuscript.

Funding The ICP-OES instrument used in this research was purchased with Mexico's Consejo Nacional de Ciencia y Tecnología (Conacyt) Infrastructure Grant \#268296 in 2016. JVP was supported by Conacyt Ph.D. fellowship \#426510. Experiments were funded from Cinvestav's internal budget. The SR laboratory is supported through a Ramanujan Fellowship, SERB-India (CRG/2018/002518).

Data availability The ICP-OES raw data and digital images from the protein gels were stored locally and are freely available on request. Unfortunately, the fly strains used in this study were disposed during the Covid-19 contingency.

\section{Compliance with ethical standards}

Conflict of interest The authors declare no conflicts of interest or competing interests.

\section{References}

Bettedi L, Aslam MF, Szular J, Mandilaras K, Missirlis F (2011) Iron depletion in the intestines of Malvolio mutant flies does not occur in the absence of a multicopper oxidase. J Exp Biol 214:971-978. https://doi.org/10.1242/ jeb.051664

Binggeli O, Neyen C, Poidevin M, Lemaitre B (2014) Prophenoloxidase activation is required for survival to microbial infections in Drosophila. PLoS Pathog 10:e1004067. https://doi.org/10.1371/journal.ppat.1004067

Binks T, Lye JC, Camakaris J, Burke R (2010) Tissue-specific interplay between copper uptake and efflux in Drosophila Journal of biological inorganic chemistry: JBIC: a publication of the society of biological. Inorg Chem 15:621628. https://doi.org/10.1007/s00775-010-0629-y

Chapman RF (1998) The insects: structure and function, 4th edn. Cambridge University Press, Cambridge

Comstra HS et al (2017) The interactome of the copper transporter ATP7A belongs to a network of neurodevelopmental and neurodegeneration factors. Elife 6:e24722. https:// doi.org/10.7554/eLife. 24722

Davis MM, O'Keefe SL, Primrose DA, Hodgetts RB (2007) A neuropeptide hormone cascade controls the precise onset of post-eclosion cuticular tanning in Drosophila melanogaster. Development 134:4395-4404. https://doi.org/ 10.1242/dev.009902

Dudzic JP, Kondo S, Ueda R, Bergman CM, Lemaitre B (2015) Drosophila innate immunity: regional and functional specialization of prophenoloxidases. BMC Biol 13:81. https://doi.org/10.1186/s12915-015-0193-6

Gibbs AG (1999) Laboratory selection for the comparative physiologist. J Exp Biol 202:2709-2718 
Gibbs AG, Rajpurohit S (2010) Cuticular lipids and water balance. In: Blomquist GJ, Bagneres A-G (eds) Insect hydrocarbons-biology, biochemistry, and chemical biology. Cambridge University Publisher, Cambridge, UK, pp 100-119

Gibbs AG, Chippindale AK, Rose MR (1997) Physiological mechanisms of evolved desiccation resistance in Drosophila melanogaster. J Exp Biol 200:1821-1832

Gibert JM, Peronnet F, Schlotterer C (2007) Phenotypic plasticity in Drosophila pigmentation caused by temperature sensitivity of a chromatin regulator network. PLoS Genet 3:e30. https://doi.org/10.1371/journal.pgen.0030030

Gonzalez-Morales N, Mendoza-Ortiz MA, Blowes LM, Missirlis F, Riesgo-Escovar JR (2015) Ferritin is required in multiple tissues during Drosophila melanogaster development. PLoS ONE 10:e0133499. https://doi.org/10.1371/ journal.pone.0133499

Graubard MA (1933) Tyrosinase in mutants of Drosophila melanogaster. J Genet 27:199-218

Gutierrez L, Zubow K, Nield J, Gambis A, Mollereau B, Lazaro FJ, Missirlis F (2013) Biophysical and genetic analysis of iron partitioning and ferritin function in Drosophila melanogaster. Metallomics 5:997-1005. https:// doi.org/10.1039/c3mt00118k

Hackman RH, Goldberg M (1971) Studies on the hardening and darkening of insect cuticles. J Insect Physiol 17:335347. https://doi.org/10.1016/0022-1910(71)90218-6

Hernandez-Gallardo AK, Missirlis F (2020) Loss of ferritin in developing wing cells: apoptosis and ferroptosis coincide. PLoS Genet 16:e1008503. https://doi.org/10.1371/journal. pgen.1008503

Kalmus H (1941) The resistance to desiccation of Drosophila mutants affecting body colour. Proc R Soc Lond B 130:185-201. https://doi.org/10.1098/rspb.1941.0011

Kapun M et al (2020) Genomic analysis of European Drosophila melanogaster populations reveals longitudinal structure, continent-wide selection, and previously unknown DNA viruses. Mol Biol Evol. https://doi.org/10.1093/molbev/msaa120

Kirby K, Jensen LT, Binnington J, Hilliker AJ, Ulloa J, Culotta VC, Phillips JP (2008) Instability of superoxide dismutase 1 of Drosophila in mutants deficient for its cognate copper chaperone. J Biol Chem 283(51):35393-35401. https:// doi.org/10.1074/jbc.M807131200

Lewis HW, Lewis HS (1963) Genetic regulation of dopa oxidase activity in Drosophila. Ann N Y Acad Sci 100:827839. https://doi.org/10.1111/j.1749-6632.1963.tb42934.x

Luan $\mathrm{H}$ et al (2006) Functional dissection of a neuronal network required for cuticle tanning and wing expansion in Drosophila. J Neurosci 26:573-584. https://doi.org/10. 1523/JNEUROSCI.3916-05.2006

Massey JH, Wittkopp PJ (2016) The genetic basis of pigmentation differences within and between Drosophila species. Curr Top Dev Biol 119:27-61. https://doi.org/10.1016/bs. ctdb.2016.03.004

Massey JH, Akiyama N, Bien T, Dreisewerd K, Wittkopp PJ, Yew JY, Takahashi A (2019a) Pleiotropic effects of ebony and tan on pigmentation and cuticular hydrocarbon composition in Drosophila melanogaster. Front Physiol 10:518. https://doi.org/10.3389/fphys.2019.00518
Massey JH, Chung D, Siwanowicz I, Stern DL, Wittkopp PJ (2019b) The yellow gene influences Drosophila male mating success through sex comb melanization. eLife. https:// doi.org/10.7554/eLife.49388

Ostrowski S, Dierick HA, Bejsovec A (2002) Genetic control of cuticle formation during embryonic development of Drosophila melanogaster. Genetics 161:171-182

Parkash R, Rajpurohit S, Ramniwas S (2008) Changes in body melanisation and desiccation resistance in highland vs. lowland populations of D. melanogaster. J Insect Physiol 54:1050-1056. https://doi.org/10.1016/j.jinsphys.2008.04. 008

Rajpurohit S, Gibbs AG (2012) Selection for abdominal tergite pigmentation and correlated responses in the trident: a case study in Drosophila melanogaster. Biol J Lin Soc 106:287-294. https://doi.org/10.1111/j.1095-8312.2012. 01870.x

Rajpurohit S, Peterson LM, Orr AJ, Marlon AJ, Gibbs AG (2016a) An Experimental evolution test of the relationship between melanism and desiccation survival in insects. PLoS ONE 11:e0163414. https://doi.org/10.1371/journal. pone. 0163414

Rajpurohit S, Richardson R, Dean J, Vazquez R, Wong G, Schmidt PS (2016b) Pigmentation and fitness trade-offs through the lens of artificial selection. Biol Lett. https:// doi.org/10.1098/rsbl.2016.0625

Ramniwas S, Kajla B, Dev K, Parkash R (2013) Direct and correlated responses to laboratory selection for body melanisation in Drosophila melanogaster: support for the melanisation-desiccation resistance hypothesis. J Exp Biol 216:1244-1254. https://doi.org/10.1242/jeb.076166

Rempoulakis P et al (2014) Conserved metallomics in two insect families evolving separately for a hundred million years. Biometals 27:1323-1335. https://doi.org/10.1007/ s10534-014-9793-9

Riedel F, Vorkel D, Eaton S (2010) Megalin-dependent Yellow endocytosis restricts melanization in the Drosophila cuticle. Development 138(1):149-158

Rosas-Arellano A, Vasquez-Procopio J, Gambis A, Blowes LM, Steller H, Mollereau B, Missirlis F (2016) Ferritin assembly in enterocytes of Drosophila melanogaster. Int J Mol Sci 17:27. https://doi.org/10.3390/ijms17020027

Sadraie M, Missirlis F (2011) Evidence for evolutionary constraints in Drosophila metal biology. Biometals 24:679686. https://doi.org/10.1007/s10534-011-9420-y

Sellami A, Wegener C, Veenstra JA (2012) Functional significance of the copper transporter ATP7 in peptidergic neurons and endocrine cells in Drosophila melanogaster. FEBS Lett 586:3633-3638. https://doi.org/10.1016/j.febs1 et.2012.08.009

Seybold WD, Meltzer PS, Mitchell HK (1975) Phenol oxidase activation in Drosophila: a cascase of reactions. Biochem Genet 13:85-108. https://doi.org/10.1007/BF00486009

Shakhmantsir I, Massad NL, Kennell JA (2014) Regulation of cuticle pigmentation in drosophila by the nutrient sensing insulin and TOR signaling pathways. Dev Dyn 243:393401. https://doi.org/10.1002/dvdy.24080

Sugumaran M, Giglio L, Kundzicz H, Saul S, Semensi V (1992) Studies on the enzymes involved in puparial cuticle sclerotization in Drosophila melanogaster. Arch Insect 
Biochem Physiol 19:271-283. https://doi.org/10.1002/ arch.940190406

Tejeda-Guzman C, Rosas-Arellano A, Kroll T, Webb SM, Barajas-Aceves M, Osorio B, Missirlis F (2018) Biogenesis of zinc storage granules in Drosophila melanogaster. J Exp Biol. https://doi.org/10.1242/jeb.168419

Theotoki EI et al (2019) Targeting of copper-trafficking chaperones causes gene-specific systemic pathology in Drosophila melanogaster: prospective expansion of mutational landscapes that regulate tumor resistance to cisplatin. Biol Open 8:bio046961. https://doi.org/10.1242/bio.046961

Turski ML, Thiele DJ (2007) Drosophila Ctr1A functions as a copper transporter essential for development. J Biol Chem 282:24017-24026. https://doi.org/10.1074/jbc.M7037 92200

Vasquez-Procopio J et al (2020) Intestinal response to dietary manganese depletion in Drosophila. Metallomics 12:218240. https://doi.org/10.1039/c9mt00218a

Walter MF, Zeineh LL, Black BC, McIvor WE, Wright TR, Biessmann H (1996) Catecholamine metabolism and in vitro induction of premature cuticle melanization in wild type and pigmentation mutants of Drosophila melanogaster. Arch Insect Biochem Physiol 31:219-233. https://doi.org/10.1002/(SICI)1520-6327(1996)31:2<219: AID-ARCH9>3.0.CO;2-U

Wang X, Yin S, Yang Z, Zhou B (2018) Drosophila multicopper oxidase 3 is a potential ferroxidase involved in iron homeostasis. Biochim Biophys Acta 1862(8):1826-1834. https://doi.org/10.1016/j.bbagen.2018.04.017

Wittkopp PJ, Beldade P (2009) Development and evolution of insect pigmentation: genetic mechanisms and the potential consequences of pleiotropy. Semin Cell Dev Biol 20:6571. https://doi.org/10.1016/j.semcdb.2008.10.002

Wittkopp PJ, Vaccaro K, Carroll SB (2002) Evolution of yellow gene regulation and pigmentation in Drosophila. Curr Biol 12:1547-1556. https://doi.org/10.1016/s09609822(02)01113-2

Wright TR (1987) The genetics of biogenic amine metabolism, sclerotization, and melanization in Drosophila melanogaster. Adv Genet 24:127-222

Xiao G, Wan Z, Fan Q, Tang X, Zhou B (2014) The metal transporter ZIP13 supplies iron into the secretory pathway in Drosophila melanogaster. eLife 3:e03191. https://doi. org/10.7554/eLife.03191

Zhang B, Binks T, Burke R (2020) The E3 ubiquitin ligase $\mathrm{Slimb} / \beta-\operatorname{TrCP}$ is required for normal copper homeostasis in Drosophila. Biochim Biophys Acta Mol Cell Res. https://doi.org/10.1016/j.bbamcr.2020.118768

Zhou H, Cadigan KM, Thiele DJ (2003) A copper-regulated transporter required for copper acquisition, pigmentation, and specific stages of development in Drosophila melanogaster. J Biol Chem 278:48210-48218. https://doi.org/ 10.1074/jbc.M309820200

Publisher's Note Springer Nature remains neutral with regard to jurisdictional claims in published maps and institutional affiliations. 